\title{
Síndrome de intestino irritable: la importancia de los antiespasmódicos
}

\section{Irritable bowel syndrome. Relevance of antispasmodics}

Luis M. Bustos-Fernández, MD..$^{*}$

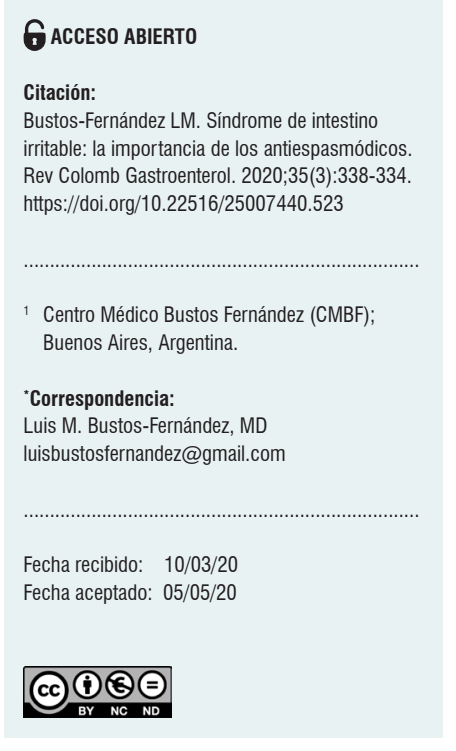

\begin{abstract}
Resumen
El síndrome de intestino irritable se caracteriza por la existencia de dolor abdominal relacionado con cambios en el ritmo evacuatorio. A pesar de los avances en el conocimiento de su fisiopatología y de la aparición de nuevas formas terapéuticas, los antiespasmódicos se han mantenido en el tiempo como una forma efectiva para el manejo de los síntomas de este síndrome, en especial para el dolor. Así pues, el propósito de esta revisión es la búsqueda de evidencia científica que soporte el uso de antiespasmódicos en el manejo de los síntomas del síndrome de intestino irritable.
\end{abstract}

\section{Palabras clave}

Síndrome de intestino irritable, antiespasmódicos, dolor abdominal.

\begin{abstract} of irritable bowel symptoms.

\section{Keywords}

Irritable bowel syndrome; Antispasmodic; Abdominal pain.
\end{abstract}

Irritable bowel syndrome is a disorder characterized by abdominal pain related to changes in bowel movements. Despite the progress made in the knowledge of its pathophysiology and the emergence of new therapeutic forms, antispasmodics have remained over time as an effective way to treat symptoms, especially pain. The purpose of this review is to search for scientific evidence on the use of antispasmodics in the treatment

\section{INTRODUCCIÓN}

Los trastornos intestinales funcionales (TIF) se caracterizan por la aparición de síntomas predominantes como el dolor abdominal, la hinchazón, distensión o anormalidades del hábito intestinal (por ejemplo, constipación, diarrea o una mezcla de estos). Los TIF se pueden distinguir de otros trastornos gastrointestinales por la cronicidad (6 meses de síntomas en el momento de presentación), la actividad actual (síntomas presentes dentro los últimos 3 meses), la frecuencia (síntomas presentes, en promedio, al menos 1 día por semana) y la ausencia de anomalías anatómicas o fisiológicas obvias, identificadas por exámenes de diagnóstico de rutina, según se considere clínicamente apropiado (1).

Los TIF se clasifican en 5 categorías distintas:

- El síndrome de intestino irritable (SII).

- Constipación funcional (CF).

- Diarrea funcional (DF).

- Distensión abdominal funcional (DAF).

- Molestias inespecíficas.

El SII es la más frecuente de estas dolencias y su prevalencia mundial es del 11,2\%. Dichos datos se basan en un metaanálisis de 80 estudios, con 260960 sujetos evaluados. Las tasas 
de prevalencia son más altas en las mujeres que en los hombres, mientras que las personas más jóvenes son más propensas a ser afectadas que aquellos mayores de 50 años (2).

Dentro de este contexto, el SII es un trastorno multifactorial, con una fisiopatología compleja. Los factores que aumentan el riesgo de desarrollar este síndrome pueden ser genéticos, ambientales o psicosociales, y los aspectos que desencadenan el inicio o la exacerbación de los síntomas pueden incluir una gastroenteritis previa, intolerancias alimentarias y estrés crónico (3).

Entre tanto, los mecanismos fisiopatológicos son variables y distintos en cada individuo. En ellos se pueden incluir una motilidad alterada, hiperalgesia visceral, aumento de la permeabilidad intestinal, activación inmune, microbiota alterada y alteraciones del eje intestino-cerebro.

De otro lado, se han asociado distintas alteraciones psicológicas con el SII $(4,5)$. Las molestias más frecuentes son angustia, depresión, vulnerabilidad afectiva y trastornos de autoestima (6). En ese sentido, el tratamiento del SII comienza por brindar información sobre las características del síndrome, para lo cual se proporciona seguridad en cuanto a la historia natural benigna y se educa al paciente sobre la utilidad y la seguridad de las distintas pruebas diagnósticas.

El tratamiento se plantea de acuerdo con el tipo de síntomas y la gravedad de ellos. Las modificaciones en el estilo de vida pueden mejorar dichos síntomas. Entre estos cambios, se incluyen realizar actividad física, reducir el estrés y mejorar la calidad del sueño (7). Se han propuesto distintos tratamientos para el manejo de este síndrome, los cuales se basan en el control de sus síntomas principales.

- Diarrea:

- Agonistas opioides (loperamida) (8).

- Dieta (baja en oligosacáridos, disacáridos, monosacáridos y polioles fermentables [Fermentable Oligosaccharides, Disaccharides, Monosaccharides And Polyols, FODMAP], y baja o libre de gluten) (9-11).

- Secuestrantes de sales biliares (colestiramina) (12).

- Probióticos/antibióticos (rifaximina) (13-15).

- Constipación:

- Psyllium (16).

- Polietilenglicol (PEG) (17).

- Agonistas de canales de cloro (linaclotida, lubiprostona) $(18,19)$.

- Procinéticos (20).

- Dolor abdominal:

- Antiespasmódicos (butilbromuro de hioscina) (16).

- Reguladores del músculo liso (21).

- Antidepresivos tricíclicos (amitriptilina) (22).
- Inhibidores selectivos de recaptación de serotonina (ISRS) (paroxetina, escitalopram) (22).

\section{ANTIESPASMÓDICOS}

Los antiespasmódicos pertenecen a un grupo de fármacos que actúan sobre el músculo liso intestinal. Estas sustan-

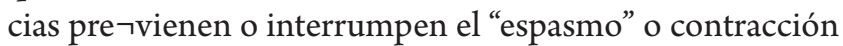
dolorosa del mismo (44). Este es uno de los mecanismos que generan el síntoma doloroso en el síndrome de intestino irritable (23). De acuerdo con su mecanismo de acción, se pueden clasificar así:

- Agentes relajantes directos del músculo liso (mebeverina, agentes derivados de la papaverina).

- Agentes anticolinérgicos (butilbromuro de hioscina, hioscina, hiosciamina, levofloxacina, dicicloverina, butilescopolamina, trimebutina y bromuro de cimetropio).

- Agentes bloqueadores de los canales del calcio (bromuro de pinaverio, bromuro de otilonio, alverina, fenoverina, rociverina y pirenzepina).

Los relajantes directos del músculo liso reducen el tono y el peristaltismo. Es así como pueden aliviar el dolor intestinal sin afectar de forma sustancial la motilidad gastrointestinal. Por otra parte, los antiespasmódicos anticolinérgicos atenúan los espasmos o las contracciones en el intestino y, por tanto, tienen el potencial de reducir el dolor abdominal $(23,24)$. Entre tanto, los antagonistas del calcio relajan el intestino al prevenir la entrada de este en las células del músculo liso intestinal. Dado que el calcio desencadena la cascada de sucesos que activa la contracción muscular, su inhibición en las células causa relajación intestinal. Este grupo de medicamentos, al reducir el índice de motilidad, puede disminuir el reflejo gastrocólico y modificar el tiempo de tránsito colónico.

El N-butilbromuro de hioscina es el antiespasmódico más expandido en el mercado, tanto como venta libre o bajo fórmula médica. Se comercializa como una monodroga o un asociado a los analgésicos. Este es un alcaloide derivado de la belladona, cuyas propiedades farmacológicas se deben a sus efectos anticolinérgicos, que lo hacen efectivo en la disminución de la frecuencia y la intensidad de los movimientos de tipo espasmódicos en el tracto gastrointestinal.

Se cree que la capacidad de este medicamento para prevenir vómitos y náuseas provocados por el movimiento está asociada a la inhibición vestibular que ejerce en el sistema nervioso central (SNC). Esto resulta en una inhibición del reflejo del vómito. Además, tiene una acción directa sobre el centro del vómito, que se encuentra en la formación reticular del tallo cerebral. Esta última acción lo plantea como una alternativa en la dispepsia funcional (25). 


\section{ANTIESPASMÓDICOS EN EL SII}

Se han realizado distintas revisiones y metaanálisis para la evaluación del manejo del SII. En 2014, el American College of Gastroenterology publicó una revisión en la que se describe que los antiespasmódicos se han utilizado durante décadas para el tratamiento del SII. Dicho trabajo se basó en el concepto según el cual muchos de los síntomas del SII corresponden al espasmo del músculo liso del colon (16).

Así, se identificaron 25 estudios controlados que evaluaron a 2154 pacientes. Solo 3 de estos trabajos usaron criterios diagnósticos estandarizados (Roma I, II) (26-28). Los otros estudios son previos a la publicación de los criterios de Roma. Esta revisión muestra que la terapia antiespasmódica tiene un efecto estadísticamente significativo en la mejora de los síntomas del SII, con un número de pacientes que es necesario tratar (Number Needed to Treat, NNT) de 5.

Sin embargo, el efecto de los antiespasmódicos en forma individual es variable y difícil de interpretar, ya que solo existe un pequeño número de estudios que evalúan cada uno de los 12 fármacos diferentes disponible para revisión. Con respecto a los agentes individuales, el bromuro de otilonio (26-28), el butilbromuro de hioscina (29-31), el bromuro de cimetropio (32-34), el bromuro de pinaverio (35) y el clorhidrato de diciclomina (36) mostraron efectos beneficiosos con unos NNT de 5, 3, 3, 3 y 4, respectivamente.

En efecto, un metaanálisis evaluó la efectividad de los agentes relajantes del músculo liso en el SII y concluyó que estos medicamentos son más efectivos que el placebo en el tratamiento de dicho síndrome (21).

En una reciente revisión sobre el tema tratamiento del SII, se evaluaron otros reguladores del musculo liso (45).

\section{OTROS REGULADORES DEL MÚSCULO LISO}

\section{Mebeverina}

La mebeverina es un antiespasmódico con acción musculotrópica, que actúa directamente sobre el músculo liso del intestino, por lo que no se asocia con efectos adversos anticolinérgicos. Los resultados en ensayos clínicos aleatorizados individuales muestran una discordancia entre ellos. Sin embargo, al analizar los datos disponibles en forma de metaanálisis no se observan diferencias estadísticamente significativas en favor de este medicamento en relación con una mejoría global de los síntomas: distensión o dolor abdominal $(21,37)$.

\section{Bromuro de otilonio}

El bromuro de otilonio es un antiespasmódico con acción sobre los receptores muscarínicos. Además, es un blo- queante de los canales de calcio, que ha sido ampliamente usado en el tratamiento del SII. En un metaanálisis publicado en 2012 se agrupan 4 ensayos clínicos aleatorizados que evaluaron el bromuro de otilonio frente a placebo. Se observan diferencias estadísticamente significativas en favor de dicho medicamento con respecto a la mejoría global de los síntomas, con un NNT de 7 y dolor abdominal con un NNT de 8. Los efectos adversos del bromuro de otilonio fueron similares a los del grupo placebo $(21,38)$.

\section{Citrato de alverina}

El citrato de alverina es un antiespasmódico bloqueante de los canales de calcio, que comúnmente se asocia con simeticona para el tratamiento del SII. Se han publicado varios ensayos clínicos aleatorizados que evalúan su acción frente a placebo o frente a tratamientos convencionales. En ellos se evidencian diferencias estadísticamente significativas en favor de este agente en cuanto a la mejoría global de síntomas y el dolor abdominal, los cuales reportan NNT de 8 y 11 , respectivamente.

Asimismo, el citrato de alverina ha mostrado generar una mejoría estadísticamente significativa de la distensión abdominal y la calidad de vida en estudios individuales realizados en pacientes con SII. Además, parece tener un perfil de seguridad alto, con efectos adversos que no difieren de los grupos placebo $(38,39)$.

\section{Bromuro de pinaverio}

El bromuro de pinaverio también es un antiespasmódico bloqueante de los canales de calcio, usado para el tratamiento del SII. Suele administrarse solo o en combinaciones. En un metaanálisis realizado por Cochrane en 2011 se agrupan 4 ensayos clínicos aleatorizados, que evaluaron la mejoría global de los síntomas del SII en pacientes tratados con bromuro de pinaverio. Se obtuvieron diferencias estadísticamente significativas en favor del grupo de tratamiento.

En 3 de estos ensayos clínicos aleatorizados se evaluó la eficacia del medicamento en la mejoría del dolor abdominal, con diferencias significativas en favor del pinaverio. Otro metaanálisis, publicado un año después, valoró los beneficios del pinaverio asociado con simeticona en 2 ensayos clínicos aleatorizados. Uno de ellos mostró beneficios significativos de dicha combinación, en relación con la mejoría de la distensión abdominal, mientras que en el otro no se observaron ventajas frente a placebo en la mejoría global de los síntomas y el dolor abdominal.

Así, tanto solo como en combinación, el bromuro de otilonio parece ser un medicamento seguro, cuyos efectos adversos no difieren de los de placebo $(21,37,38)$. 


\section{Maleato de trimebutina}

La trimebutina es un antiespasmódico bloqueante de los canales de calcio, el cual se emplea en el tratamiento del SII. En altas concentraciones, este medicamento reduce la amplitud de las contracciones espontáneas y de los potenciales de acción. También tiene un efecto analgésico y actúa como agonista débil de los receptores $\mu$ (ROM). Un metaanálisis publicado en 2001 muestra diferencias estadísticamente significativas en favor de la trimebutina en la mejoría global de los síntomas.

Entre tanto, otro metaanálisis, previamente citado en esta revisión, describe ventajas significativas de la trimebutina para el dolor abdominal. Sin embargo, los estudios con este fármaco han sido realizados con un número bajo de pacientes, lo cual explica que, al incluir o excluir alguna publicación para el análisis, difieran las conclusiones de los distintos metaanálisis.

Respecto de los efectos adversos, no se reportan diferencias frente a placebo en ninguna de las publicaciones evaluadas para esta revisión (17-19).

\section{Aceite de menta (peppermint oil)}

El aceite de menta actúa a través de varios mecanismos: es bloqueante de los canales de calcio, antagonista de los receptores $\kappa(\mathrm{ROK})$ y antagonista de los receptores 5-HT3. Por esta razón, además de tener propiedades antiespasmódicas, produce otros efectos relevantes en el tratamiento del SII, como la normalización del tiempo de tránsito orocecal (40).

La evidencia disponible sobre su utilización en el tratamiento del SII parece más sólida que la de los antiespasmódicos convencionales. Así se reporta en un metaanálisis en el que se observa una mejoría significativa del dolor abdominal y los síntomas globales, con un NNT de 4 y 3 , respectivamente. En esta revisión se reportaron efectos adversos como náuseas, vómitos y pirosis. Este último parece disminuir cuando el fármaco es administrado en tabletas con triple cubierta $(40,41)$.

\section{Eluxadolina}

La eluxadolina es un agonista de los receptores opioides $\mu$ у $\kappa$, así como también es un antagonista del receptor $\delta$ (ROD) del sistema nervioso entérico. La acción agonista de los ROM promueve el retardo del vaciamiento gástrico, el enlentecimiento del tránsito de intestinal y el aumento de la presión del esfínter anal.

De otro lado, el efecto antagonista de los ROD contrarresta la constipación resultante y aumenta la analgesia del agonismo de los ROM (42). La eluxadolina se ha indicado en el tratamiento del SII con diarrea y ha mostrado NNT que oscilan entre 8 y 33 , con valores promedio de 12,5.

En un metaanálisis de 2017, que incluyó a 2427 pacientes, se observó una mejoría estadísticamente significativa en el alivio del dolor abdominal, en la consistencia de las evacuaciones, en los síntomas globales y en la calidad de vida relacionada con el SII.

Sin embargo, se reportaron efectos adversos significativos, que incluyeron náuseas, vómitos, dolor abdominal y constipación. También se encontró una mayor incidencia de pancreatitis y de espasmo del esfínter de Oddi, en particular en pacientes previamente colecistectomizados. Debido a la aparición de estos eventos, la FDA (Food and Drug Administration) contraindica el uso de la eluxadolina en personas con historia de trastornos biliares, pancreatitis, insuficiencia hepática grave o alcoholismo, así como en aquellos individuos colecistectomizados $(42,43)$.

\section{TRATAMIENTOS DEL SII SEGÚN LA WGO}

En 2016, la Organización Mundial de Gastroenterología (World Gastroenterology Organisation, WGO) publicó la última revisión de la guía sobre SII. En ella se abordan temas con respecto al tratamiento de este síndrome y se proponen unas opciones terapéuticas de acuerdo con las distintas posibilidades económicas de la población (44).

\section{Bajos niveles de recursos}

- Medidas generales: brindar información, tranquilizar, revisar la dieta y el estilo de vida.

- Tratamiento sintomático:

- Dolor: antiespasmódico disponible localmente.

- Constipación: medidas dietéticas y suplementos de fibra.

- Diarrea: agentes formadores de masa y loperamida.

\section{Medianos niveles de recursos}

- Se agrega un probiótico de calidad con eficacia comprobada.

\section{Altos niveles de recursos}

- Se pueden agregar psicofármacos, tratamientos psicológicos, consultas con dietistas especializados y agentes farmacológicos específicos (lubiprostona, linaclotida, rifaximina).

Estos datos dejan en claro que el grupo revisor de estas guías propone la utilización de los antiespasmódicos de mayor disponibilidad local como primera línea de tratamiento (44). 


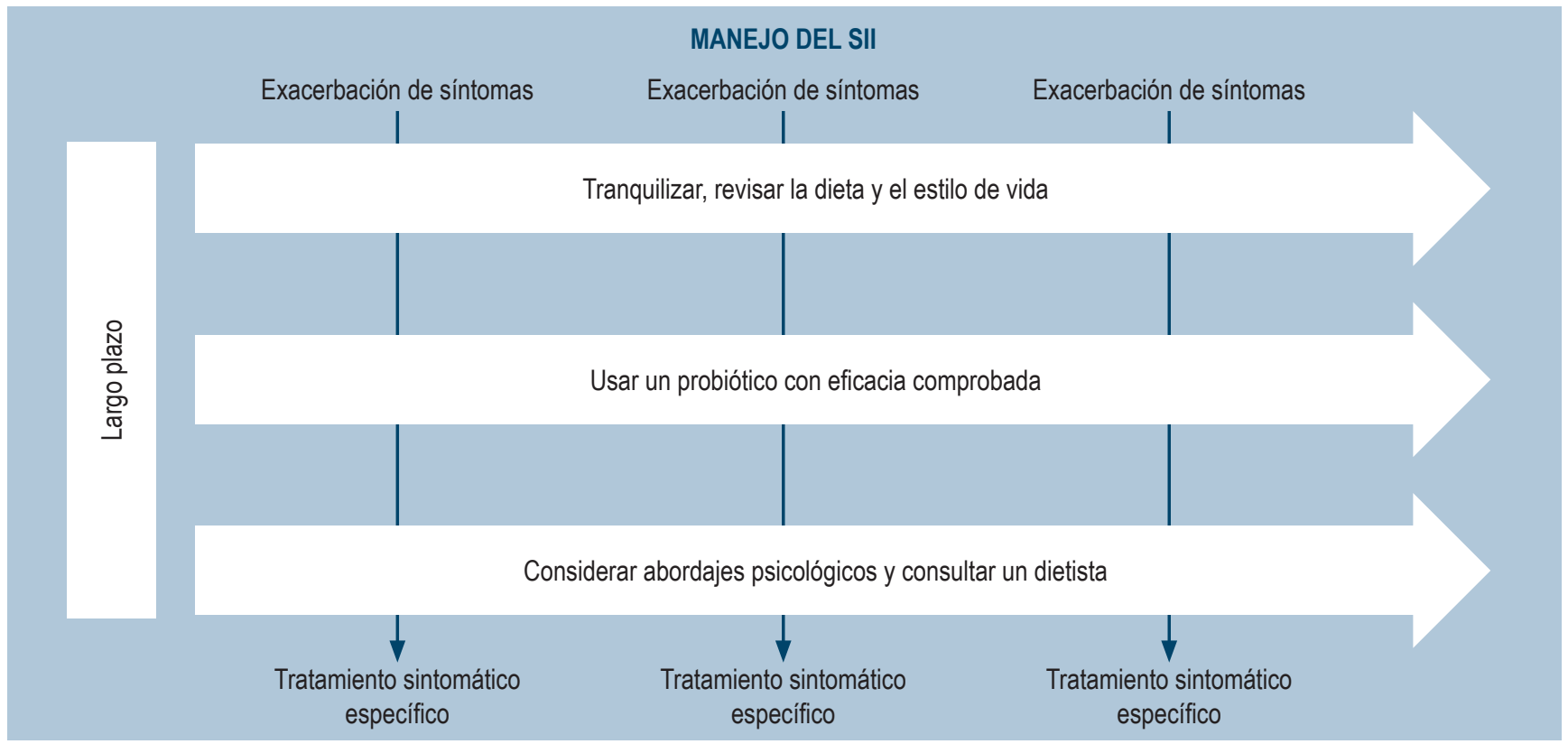

Figura 1. Manejo del SII. SII: síndrome de intestino irritable.

Así pues, la guía propone un esquema para el manejo del SII, en el cual plantea un tratamiento sintomático específico de acuerdo con el tipo y la intensidad del síntoma, así como con la disponibilidad del fármaco (Figura 1).

De otro lado, las guías de la Asociación Americana de Gastroenterología (American Gastroenterological Asso-ciation, AGA) revelan una significativa mejoría de los síntomas del SII mediante el uso de antiespasmódicos. No obstante, matizan que la utilización de estos medicamentos es limitada, ya que la mayoría no se comercializan en Estados Unidos (46).

En 2019, se publicó un último metaanálisis en el cual se evaluaron las distintas terapéuticas para el tratamiento del SII; entre ellas, se incluyeron la fibra soluble, los medicamentos antiespasmódicas y los neuromoduladores del eje intestino-cerebro. Con respecto a los antiespasmódicos, se concluyó que las evidencias que respaldan el uso del aceite de menta son las más convincentes y significativas (46).

\section{CONCLUSIONES}

El tratamiento del SII supone distintos niveles de complejidad. En esa medida, los antiespasmódicos continúan ganándose un espacio importante para el manejo de dicho síndrome, respaldados por diversas evidencias científicas. Más allá de que un mayor conocimiento de la fisiopatología de la enfermedad ha permitido encontrar nuevas opciones terapéuticas, la utilización de estos medicamentos sigue teniendo vigencia como tratamiento sintomático específico.

\section{REFERENCIAS}

1. Lacy BE, Mearin F, Chan L, Chey WD, Lembo AJ, Simren M, Spiller R. Bowel Disorders. Gastroenterology. 2016;150(6):1393-1407. http://doi.org/10.1053/j.gastro.2016.02.031

2. Lovell RM, Ford AC. Global prevalence of and risk factors for irritable bowel syndrome: a meta-analysis. Clin Gastroenterol Hepatol. 2012;10(7):712-721.e4. http://doi.org/10.1016/j.cgh.2012.02.029

3. Camilleri M, Lasch $\mathrm{K}$, Zhou W. Irritable bowel syndrome: methods, mechanisms, and pathophysiology. The con- fluence of increased permeability, inflammation, and pain in irritable bowel syndrome. Am J Physiol Gastrointest Liver Physiol. 2012;303(7):G775-G785. http://doi.org/10.1152/ajpgi.00155.2012

4. Drossman DA, McKee DC, Sandler RS, Mitchell CM, Cramer EM, Lowman BC, Burger AL. Psychosocial factors in the irritable bowel syndrome. A multivariate study of patients and nonpatients with irritable bowel syndrome. Gastroenterology. 1988;95(3):701-8. http://doi.org/10.1016/s0016-5085(88)80017-9 
5. Blewett A, Allison M, Calcraft B, Moore R, Jenkins P, Sullivan G. Psychiatric disorder and outcome in irritable bowel syndrome. Psychosomatics. 1996;37(2):155-160. http://doi.org/10.1016/S0033-3182(96)71582-7

6. Drossman DA. Do psychosocial factors define symptom severity and patient status in irritable bowel syndrome? Am J Med. 1999; 107(5A):41S-50S. http://doi.org/10.1016/s0002-9343(99)00081-9

7. Johannesson E, Simrén M, Strid H, Bajor A, Sadik R. Physical activity improves symptoms in irritable bowel syndrome: a randomized controlled trial. Am J Gastroenterol. 2011;106(5):915-922. http://doi.org/10.1038/ajg.2010.480

8. Rao AS, Wong BS, Camilleri M, Odunsi-Shiyanbade ST, McKinzie S, Ryks M, Burton D, Carlson P, Lamsam J, Singh $\mathrm{R}$, Zinsmeister AR. Chenodeoxycholate in females with irritable bowel syndrome-constipation: a pharmacodynamic and pharmacogenetic analysis. Gastroenterology. 2010;139(5):1549-58, 1558.e1.

http://doi.org/10.1053/j.gastro.2010.07.052

9. Biesiekierski JR, Newnham ED, Irving PM, Barrett JS, Haines M, Doecke JD, Shepherd SJ, Muir JG, Gibson PR. Gluten causes gastrointestinal symptoms in subjects without celiac disease: a double-blind randomized placebo-controlled trial. Am J Gastroenterol. 2011;106(3):508-14; quiz 515. http://doi.org/10.1038/ajg.2010.487

10. Vázquez-Roque MI, Camilleri M, Smyrk T, Murray JA, Marietta E, O'Neill J, Carlson P, Lamsam J, Janzow D, Eckert D, Burton D, Zinsmeister AR. A controlled trial of gluten-free diet in patients with irritable bowel syndromediarrhea: effects on bowel frequency and intestinal function. Gastroenterology. 2013;144(5):903-911. http://doi.org/10.1053/j.gastro.2013.01.049

11. Moayyedi P, Quigley EM, Lacy BE, Lembo AJ, Saito YA, Schiller LR, Soffer EE, Spiegel BM, Ford AC. The Effect of Dietary Intervention on Irritable Bowel Syndrome: A Systematic Review. Clin Transl Gastroenterol. 2015;6(8):e107. http://doi.org/10.1038/ctg.2015.21

12. Wong BS, Camilleri M, Carlson P, McKinzie S, Busciglio I, Bondar O, Dyer RB, Lamsam J, Zinsmeister AR. Increased bile acid biosynthesis is associated with irritable bowel syndrome with diarrhea. Clin Gastroenterol Hepatol. 2012;10(9):1009-15. http://doi.org/10.1016/j.cgh.2012.05.006

13. Ford AC, Quigley EM, Lacy BE, Lembo AJ, Saito YA, Schiller LR, Soffer EE, Spiegel BM, Moayyedi P. Efficacy of prebiotics, probiotics, and synbiotics in irritable bowel syndrome and chronic idiopathic constipation: systematic review and meta-analysis. Am J Gastroenterol. 2014;109(10):1547-61. http://doi.org/10.1038/ajg.2014.202

14. Pimentel M, Lembo A, Chey WD, Zakko S, Ringel Y, Yu J, Mareya SM, Shaw AL, Bortey E, Forbes WP; TARGET Study Group. Rifaximin therapy for patients with irritable bowel syndrome without constipation. $\mathrm{N}$ Engl J Med.
2011;364(1):22-32.

http://doi.org/10.1056/NEJMoa1004409

15. Lembo A, Pimentel M, Rao SS, Schoenfeld P, Cash B, Weinstock LB, Paterson C, Bortey E, Forbes WP. Repeat Treatment With Rifaximin Is Safe and Effective in Patients With Diarrhea-Predominant Irritable Bowel Syndrome. Gastroenterology. 2016;151(6):1113-1121. http://doi.org/10.1053/j.gastro.2016.08.003

16. Ford AC, Moayyedi P, Lacy BE, Lembo AJ, Saito YA, Schiller LR, Soffer EE, Spiegel BM, Quigley EM; Task Force on the Management of Functional Bowel Disorders. American College of Gastroenterology monograph on the management of irritable bowel syndrome and chronic idiopathic constipation. Am J Gastroenterol. 2014;109 Suppl 1:S2-26. http://doi.org/10.1038/ajg.2014.187

17. Chapman RW, Stanghellini V, Geraint M, Halphen M. Randomized clinical trial: macrogol/PEG 3350 plus electrolytes for treatment of patients with constipation associated with irritable bowel syndrome. Am J Gastroenterol. 2013;108(9):1508-1515. http://doi.org/10.1038/ajg.2013.197

18. Drossman DA, Chey WD, Johanson JF, Fass R, Scott C, Panas R, Ueno R. Clinical trial: lubiprostone in patients with constipation-associated irritable bowel syndrome-results of two randomized, placebo-controlled studies. Aliment Pharmacol Ther. 2009;29(3):329-41. http://doi.org/10.1111/j.1365-2036.2008.03881.x

19. Chey WD, Drossman DA, Johanson JF, Scott C, Panas RM, Ueno R. Safety and patient outcomes with lubiprostone for up to 52 weeks in patients with irritable bowel syndrome with constipation. Aliment Pharmacol Ther. 2012;35(5):587-599. http://doi.org/10.1111/j.1365-2036.2011.04983.x

20. Omer A, Quigley EMM. An update on prucalopride in the treatment of chronic constipation. Therap Adv Gastroenterol. 2017;10(11):877-887. http://doi.org/10.1177/1756283X17734809

21. Poynard T, Regimbeau C, Benhamou Y. Meta-analysis of smooth muscle relaxants in the treatment of irritable bowel syndrome. Aliment Pharmacol Ther. 2001;15(3):355-361. http://doi.org/10.1046/j.1365-2036.2001.00937.x

22. Ford AC, Quigley EM, Lacy BE, Lembo AJ, Saito YA, Schiller LR, Soffer EE, Spiegel BM, Moayyedi P. Effect of antidepressants and psychological therapies, including hypnotherapy, in irritable bowel syndrome: systematic review and meta-analysis. Am J Gastroenterol. 2014;109(9):1350-65. http://doi.org/10.1038/ajg.2014.148

23. Spiller R, Aziz Q Creed F, Emmanuel A, Houghton L, Hungin P, Jones R, Kumar D, Rubin G, Trudgill N, Whorwell P; Clinical Services Committee of The British Society of Gastroenterology. Guidelines on the irritable bowel syndrome: mechanisms and practical management. Gut. 2007;56(12):1770-98. http://doi.org/10.1136/gut.2007

24. Lembo T, Rink R. Current pharmacologic treatments of irritable bowel syndrome. participate (international foun- 
dation for functional gastrointestinal disorders). $\mathrm{N}$ Engl J Med 2002;11:1-4.

25. Bouin M, Lupien F, Riberdy-Poitras M, Poitras P. Tolerance to gastric distension in patients with functional dyspepsia: modulation by a cholinergic and nitrergic method. Eur J Gastroenterol Hepatol. 2006;18(1):63-68. http://doi.org/10.1097/00042737-200601000-00011

26. Clavé P, Acalovschi M, Triantafillidis JK, Uspensky YP, Kalayci C, Shee V, Tack J; OBIS Study Investigators. Randomised clinical trial: otilonium bromide improves frequency of abdominal pain, severity of distention and time to relapse in patients with irritable bowel syndrome. Aliment Pharmacol Ther. 2011;34(4):432-42. http://doi.org/10.1111/j.1365-2036.2011.04730.x

27. Glende M, Morselli-Labate AM, Battaglia G, Evangelista $S$. Extended analysis of a double-blind, placebocontrolled, 15-week study with otilonium bromide in irritable bowel syndrome. Eur J Gastroenterol Hepatol. 2002;14(12):1331-1338. http://doi.org/10.1097/00042737-200212000-00008

28. Mitchell SA, Mee AS, Smith GD, Palmer KR, Chapman RW. Alverine citrate fails to relieve the symptoms of irritable bowel syndrome: results of a double-blind, randomized, placebo-controlled trial. Aliment Pharmacol Ther. 2002;16(6):1187-1195. http://doi.org/10.1046/j.1365-2036.2002.01277.x

29. Nigam P, Kapoor KK, Rastog CK, Kumar A, Gupta AK. Different therapeutic regimens in irritable bowel syndrome. J Assoc Physicians India. 1984;32(12):1041-1044.

30. Ritchie JA, Truelove SC. Treatment of irritable bowel syndrome with lorazepam, hyoscine butylbromide, and ispaghula husk. Br Med J. 1979;1(6160):376-378. http://doi.org/10.1136/bmj.1.6160.376

31. Schäfer E, Ewe K. The treatment of irritable colon. Efficacy and tolerance of buscopan plus, buscopan, paracetamol and placebo in ambulatory patients with irritable colon. Fortschr Med. 1990;108(25):488-92.

32. Centonze V, Imbimbo BP, Campanozzi F, Attolini E, Daniotti $\mathrm{S}$, Albano O. Oral cimetropium bromide, a new antimuscarinic drug, for long-term treatment of irritable bowel syndrome. Am J Gastroenterol. 1988;83(11):1262-1266.

33. Dobrilla G, Imbimbo BP, Piazzi L, Bensi G. Longterm treatment of irritable bowel syndrome with cimetropium bromide: a double blind placebo controlled clinical trial. Gut. 1990;31(3):355-8. http://doi.org/10.1136/gut.31.3.355

34. Passaretti S, Guslandi M, Imbimbo BP, Daniotti S, Tittobello A. Effects of cimetropium bromide on gastrointestinal transit time in patients with irritable bowel syndrome. Aliment Pharmacol Ther. 1989;3(3):267-276. http://doi.org/10.1111/j.1365-2036.1989.tb00213.x

35. Virat J, Hueber D. Colopathy pain and dicetel. Prat Med. 1987;43:32-4.

36. Page JG, Dirnberger GM. Treatment of the irritable bowel syndrome with Bentyl (dicyclomine hydrochloride). J Clin
Gastroenterol. 1981;3(2):153-156.

http://doi.org/10.1097/00004836-198106000-00009

37. Martínez-Vázquez MA, Vázquez-Elizondo G, GonzálezGonzález JA, Gutiérrez-Udave R, Maldonado-Garza HJ, Bosques-Padilla FJ. Effect of antispasmodic agents, alone or in combination, in the treatment of Irritable Bowel Syndrome: systematic review and meta-analysis. Rev Gastroenterol Mex. 2012;77(2):82-90. http://doi.org/10.1016/j.rgmx.2012.04.002

38. Ruepert L, Quartero AO, de Wit NJ, van der Heijden GJ, Rubin G, Muris JW. Bulking agents, antispasmodics and antidepressants for the treatment of irritable bowel syndrome. Cochrane Database Syst Rev. 2011;(8):CD003460. http://doi.org/10.1002/14651858.CD003460.pub3

39. Ducrotte P, Grimaud JC, Dapoigny M, Personnic S, O'Mahony V, Andro-Delestrain MC. On-demand treatment with alverine citrate/simeticone compared with standard treatments for irritable bowel syndrome: results of a randomised pragmatic study. Int J Clin Pract. 2014;68(2):245-254. http://doi.org/10.1111/ijcp.12333

40. Khanna R, MacDonald JK, Levesque BG. Peppermint oil for the treatment of irritable bowel syndrome: a systematic review and meta-analysis. J Clin Gastroenterol. 2014;48(6):505-512. http://doi.org/10.1097/MCG.0b013e3182a88357

41. Cash BD, Epstein MS, Shah SM. A Novel Delivery System of Peppermint Oil Is an Effective Therapy for Irritable Bowel Syndrome Symptoms. Dig Dis Sci. 2016;61(2):560-571. http://doi.org/10.1007/s10620-015-3858-7

42. Fragkos KC. Spotlight on eluxadoline for the treatment of patients with irritable bowel syndrome with diarrhea. Clin Exp Gastroenterol. 2017; 10:229-240.

http://doi.org/10.2147/CEG.S123621

43. Harinstein L, Wu E, Brinker A. Postmarketing cases of eluxadoline-associated pancreatitis in patients with or without a gallbladder. Aliment Pharmacol Ther. 2018;47(6):809-815. http://doi.org/10.1111/apt.14504

44. Hani A. Antiespasmódicos. Guía Latinoamericana de Dispepsia Funcional. Acta Gastroenterol Latinoam. 2014;44(sup2):S57-S60.

45. Bustos Fernandez LM, Hanna-Jairala I. Tratamiento actual del síndrome de intestino irritable. Una nueva visión basada en la experiencia y la evidencia. Acta Gastroenterol Latinoam 2019;49(4):381-393

46. Quigley EM, Fried M, Gwee KA, Khalif I, Hungin AP, Lindberg G, Abbas Z, Fernandez LB, Bhatia SJ, Schmulson M, Olano C, LeMair A; Review Team: World Gastroenterology Organisation Global Guidelines Irritable Bowel Syndrome: A Global Perspective Update September 2015. J Clin Gastroenterol. 2016;50(9):704-13. http://doi.org/10.1097/MCG.0000000000000653 